\title{
Typ-2-Diabetes ist ansteckend
}

\author{
Ob jemand an Diabetes erkrankt oder nicht, hängt
}

auch von den Menschen ab, die ihm nahestehen.

US-Mediziner empfehlen, zur Abschätzung des Diabetesrisikos von Patienten auch Informationen über Ehepartner, Geschwister und Freunde zu betrachten. Seien die stark übergewichtig oder zuckerkrank, könne von einem erhöhten Erkrankungsrisiko ausgegangen werden. V.a. Ehepartner mit Adipositas und Geschwister mit Typ-2-Diabetes (T2D) sind nach einer retrospektiven Analyse der Framingham Offspring Study (FOS) Risikoindikatoren für T2D, unabhängig vom genetischen Risiko des Betreffenden.

Für die Analyse standen Daten von 4797 FOS-Teilnehmern zur Verfügung, die zwischen 1971 und 2008 zu 8 Zeitpunkten erhoben worden waren. Die Daten beinhalten Angaben zur kardiometabolischen Gesundheit sowohl der Studienteilnehmer als auch von engen Kontaktpersonen; bei den 8 Terminen wurden zwischen 9300 und 14.400 Ehepartner, Geschwister, Freunde u./o. Nachbarn erfasst. Ein T2D bestand bei 136 Teilnehmern schon zum Studienstart, bei 692 wurde er in der Studie diagnostiziert.

Wenn enge Beziehungen zu fettleibigen bzw. zu zuckerkranken Personen angegeben wurden, erhöhte dies die Wahrscheinlichkeit einer späteren Diabetesdiagnose signifikant um 32\% bzw. um 21\%. Eine Aufschlüsselung nach der Art der Beziehung er- gab, dass bei einer Adipositas des Ehepartners das Diabetesrisiko um 54\% erhöht war. Starkes Übergewicht bei Geschwistern zeigte dagegen keinen Einfluss. Umgekehrt war ein T2D bei Schwester oder Bruder, aber nicht beim Ehepartner, mit einem höheren Erkrankungsrisiko verbunden; die Risikosteigerung lag bei $64 \%$. Allein durch gemeinsame Gene war das nicht zu erklären: Das zusätzliche Risiko bestand auch noch, wenn genetische Veranlagung und Elternanamnese berücksichtigt wurden. Teilnehmer mit genetisch bedingt hohem Erkrankungsrisiko waren sogar besonders anfällig für den Effekt von Adipositas und T2D bei nahestehenden Personen. „Wir gehen davon aus, dass die Assoziationen zustande kommen, weil die Beteiligten dasselbe Gesundheitsverhalten haben, v.a. in Bezug auf Ernährung und Sport", schreiben die Studienautoren. Informationen über nahestehende Personen könnten daher dazu beitragen, Patienten mit hohem T2D-Risiko früher zu erkennen und präventive Maßnahmen einzuleiten. Die Ergebnisse sind nach Einschätzung der US-Ärzte zudem für Präventionsprogramme relevant. Damit die erfolgreich sind, sollten auch Wechselwirkungen im sozialen Umfeld Beachtung finden.

Dr. Beate Schumacher

\author{
Referenzen: \\ 1. Raghavan S et al. J Gen Intern Med 2016;31(10):1127-33
}

\section{Screening-Befund Diabetes \\ Oft schon in den Augen zu sehen}

\author{
Jeder achte Patient mit Typ-2-Diabetes, der bei ei- \\ nem allgemeinen Screening entdeckt wird, hat be- \\ reits eine diabetische Retinopathie.
}

Reihenuntersuchungen auf Diabetes könnten dazu beitragen, dass weniger Diabetiker an Sehbehinderungen leiden. Diesen Schluss ziehen Ärzte der Universitätsmedizin Mainz aus einer neuen Analyse der Gutenberg-Gesundheitsstudie. Patienten, deren Typ-2-Diabetes bei einem Screening entdeckt worden war, hatten zum Zeitpunkt der Diagnose zu 13\% bereits eine Retinopathie. Bei den meisten Netzhautschäden war allerdings noch keine Therapie erforderlich.

\section{Retinopathien alle noch asymptomatisch}

Die Studie untersucht den Gesundheitszustand der Bevölkerung in der Rhein-Main-Region, mit einem Schwerpunkt auf der Herz-Kreislauf-Gesundheit. Von 14.948 Teilnehmern im Alter zwischen 35 und 74 Jahren hatten 1377 (9,2\%) einen Diabetes mellitus, bei 347 Patienten war die Erkrankung aufgrund des Screenings erstmals aufgefallen. Die Reihenuntersuchung hatte damit bei einer von 50 Personen eine Diabetes-Neudiagnose zur Folge. 285 der neu erkannten Diabetikern waren auch ophthal- moskopisch untersucht worden. Bei 36 wurde eine Retinopathie entdeckt. 33 Patienten hatten eine leichte und zwei eine mittelschwere nichtproliferative Form, ein einzelner Patient litt bereits an einer proliferativen Retinopathie. Auswirkungen auf die Sehschärfe waren noch bei keinem der Patienten zu erkennen. Bei den meisten der Patienten mit Retinopathie (30 von 36) wurde ein zu hoher Blutdruck festgestellt. Eine Hypertonie bedeutete eine Verdopplung des Risikos für einen Netzhautschaden. Die HbA1C-Werte waren dagegen in beiden Gruppen ähnlich (im Median 6,8\%: 6,5-15,0\% mit und 6,5-13,8\% ohne Retinopathie). Gewicht und Blutfette standen ebenfalls nicht in Zusammenhang mit dem Auftreten der Mikroangiopathie. „Unsere Ergebnisse spiegeln die Tatsache wider, dass ein Diabetes selbst dann noch asymptomatisch sein kann, wenn bereits Endorganschäden vorliegen", schreiben die Studienautoren um Katharina A. Ponto. Darin zeige sich die Notwendigkeit eines Screenings auf Typ-2-Diabetes, um frühe Komplikationen zu entdecken und schwere Retino- und Makulopathien zu verhindern.

Dr. Beate Schumacher 